\title{
Postmortem serum protein growth arrest-specific 6 levels in sepsis-related deaths
}

\author{
Cristian Palmiere $^{1} \cdot$ Marc Augsburger $^{1}$
}

Received: 1 April 2015 /Accepted: 26 June 2015 / Published online: 2 August 2015

(C) Springer-Verlag Berlin Heidelberg 2015

\begin{abstract}
Growth arrest-specific 6 (Gas6) is widely expressed in leukocytes, platelets, endothelial cells, and monocytes. It regulates various processes including granulocyte adhesion to the endothelium, cell migration, thrombus stabilization, and cytokine release. In humans, increased plasma Gas6 levels have been described in patients with sepsis and septic shock. In this study, Gas 6 concentrations were measured in postmortem serum from femoral blood in a series of sepsis-related fatalities and control cases. The aims were twofold: first, to determine whether Gas6 can be reliably determined in postmortem serum; and second, to assess its diagnostic potential in identifying sepsis-related deaths. Two study groups were prospectively formed, a sepsis-related fatalities group (24 cases) and a control group (24 cases) including cases of deep vein thrombosis and fatal pulmonary embolism, cases of systemic inflammatory response syndrome in severe trauma, cases of end-stage renal failure, and cases of hanging (non-septic, nonSIRS, non-end stage renal failure cases). The preliminary results of this study seem to indicate that Gas6 can be effectively measured in postmortem serum. However, Gas6 levels in sepsis-related fatalities do not appear to be clearly distinguishable from concentrations in pulmonary embolism, severe trauma, and end-stage renal failure cases. These findings tend to support previous reports that indicated that Gas6 behaves as an acute phase reactant and can be considered a general marker of inflammation rather than a specific biomarker of sepsis.
\end{abstract}

Cristian Palmiere

cristian.palmiere@chuv.ch

1 CURML, Lausanne, Switzerland
Keywords Gas6 · Inflammation markers · Inflammation · Infection $\cdot$ Sepsis $\cdot$ Autopsy

\section{Introduction}

Protein growth arrest-specific 6 (Gas6) is a $75-\mathrm{kDa}$ protein structurally related to protein $\mathrm{S}$, a cofactor for the protein $\mathrm{C}$ anticoagulant pathway. Gas 6 belongs to the family of plasma vitamin $\mathrm{K}$-dependent proteins and is widely expressed in leukocytes and platelets as well as terminally differentiated cells in numerous organs such as vascular smooth muscle cells, endothelial cells, fibroblasts, bone marrow stromal cells, monocytes, and neurons [1-3].

Gas6 interacts with receptor tyrosine kinases of the TAM (Tyro-3, Axl, Mer) family and up- or downregulates various processes including survival and proliferation in different cell types, granulocyte adhesion to vascular endothelium, cell migration, thrombus stabilization after platelet activation, cytokine release, and protection from cellular death [2-6].

Among its numerous functions, the Gas6/TAM system appears to be involved in interactions between endothelial cells (EC), leukocytes, and platelets. Moreover, EC and leukocytes have been shown to express Gas6 and TAM receptors, especially in conditions of inflammation $[2,5,7,8]$.

The role of the Gas6/TAM system has been found to be important in innate immunity control, inflammatory disease, autoimmune disease, and cancer. Experimental research has revealed that mice lacking TAM receptors (TAM $-/-$ mice) have a high susceptibility to developing endotoxic shock (with an exaggerated elevation of tumor necrosis factor alpha (TNF- $\alpha$ ) and interleukin 6 (IL-6) production after the lipopolysaccharide (LPS) challenge), unregulated immunity, autoimmunity, and inflammation. In humans, increased plasma Gas6 levels have been described in patients with sepsis and 
septic shock, in which Gas6 concentrations appear to correlate with disease severity and increased mortality $[1-3,5,7,8]$.

Severe sepsis and septic shock remain the leading causes of death in critically ill patients in intensive care units. Hence, early identification of patients at risk for developing sepsis and septic shock continues to represent a critical step in clinical management during intensive care $[9,10]$.

In the realm of forensic pathology, the diagnosis of sepsis as the cause of death still remains challenging despite significant improvements in sample collection during postmortem examination and diagnostic strategies over recent decades. Thus, extensive efforts have been devoted during the past years to identifying the biochemical marker or the combination of biological parameters with the highest diagnostic value after death [11].

In the study herein described, Gas6 concentrations were measured in postmortem serum from femoral blood in a series of sepsis-related fatalities and control cases that underwent medicolegal investigations. Our aim was to determine whether Gas6 could be reliably determined in biological samples collected during autopsy and assess its diagnostic potential in identifying sepsis-related deaths in the postmortem setting.

\section{Materials and methods}

\section{Study design}

The present study was performed during 2010-2014 and was designed as a prospective study involving two academic hospitals. All cases collected for this study underwent medicolegal autopsies as requested by the inquiring authorities (the public prosecutor). Laboratory analyses, including Gas6 measurement, were performed as part of the medicolegal investigations.

\section{Study populations}

Two study groups were prospectively formed, a sepsis-related fatality group and a control group. The sepsis-related fatality group consisted of 24 forensic autopsy cases (16 males and 8 females between 36 and 78 years of age). No cases of neonatal sepsis were included in this group. All patients had been admitted to the intensive care unit of two academic hospitals, where they subsequently died. All cases had a documented, clinical diagnosis of sepsis in vivo (duration of sepsis between 16 and $58 \mathrm{~h}$ ).

Sepsis was diagnosed based on evidence of endocarditis, peritonitis, upper urinary tract infections, pleural empyema, pneumonia, and other respiratory infections, along with the presence of systemic inflammatory response syndrome (SIRS) according to the definition by the American College of Chest Physicians/Society of Critical Care Medicine (ACCP/SCCM) [12].

All cases included in the septic group underwent complete medicolegal autopsies. Intervals after death ranged between 12 and $48 \mathrm{~h}$.

Pleura/lung/respiratory tract infections (eight cases), endocarditis (two cases), pyelonephritis (six cases), and peritonitis following surgical anastomosis dehiscence, gastrointestinal perforation, or intraabdominal abscess rupture (eight cases) were the infectious foci identified by means of autopsy and histology.

Sepsis and multiple organ dysfunction syndrome (MODS) were postulated as the causes of death based on autopsy, histology, and biochemical investigations.

Procalcitonin (PCT), C-reactive protein (CRP), IL-6, LPS binding protein (LBP), and soluble triggering receptor expressed on myeloid cells type 1 (sTREM-1) were measured in all cases. Alternative causes of death were excluded based on further postmortem investigations.

The control group consisted of 24 age-, race-, and gendermatched forensic autopsy cases (16 males and 8 females between 35 and 76 years of age). All subjects selected for this group originated from forensic practice and included:

- Six cases of deep vein thrombosis and fatal pulmonary embolism (sudden death outside hospital in all cases).

- Six non-septic intensive care unit patients (SIRS in trauma). All these patients were admitted to hospital, where they subsequently died, with no documented, clinical diagnosis of sepsis. Diagnosis at admission was thoracic and/or abdominal trauma (blunt trauma) in all these cases. The survival time was known and within $12 \mathrm{~h}$ of major injury in all these patients.

- Six cases of end-stage renal failure (death outside hospital in all cases).

- Six non-septic, non-SIRS, non-end stage renal failure cases (death outside hospital in all cases). The cause of death was hanging in all these cases.

Intervals between deaths and autopsies ranged from 12 to $40 \mathrm{~h}$.

Histology, toxicology, microbiology, and biochemistry were performed in all sepsis and control cases. Specimens for microbiological analyses were collected from at least two different sampling sites and always included cardiac blood. Postmortem serum from femoral blood was the biological sample chosen for inflammation/infection biomarker measurement.

\section{Sample collection}

Femoral blood samples were collected from the femoral vein(s) immediately prior to autopsy. Blood was drawn after 
clamping the vein(s) at the proximal end and lifting the lower $\operatorname{limb}(\mathrm{s})$ for several minutes and subsequently collected by aspiration using a sterile needle and syringe. Blood was stored in tubes containing gel serum separator and centrifuged immediately post collection at $3000 \mathrm{~g}$ for $15 \mathrm{~min}$. After centrifugation, the separated supernatant (postmortem serum, between 1 and $5 \mathrm{ml}$ ) was collected and stored in preservative-free tubes. All samples were transferred to the laboratories immediately post collection. When analyses were delayed, samples were stored at $-20{ }^{\circ} \mathrm{C}$. No specimens were excluded due to insufficient sample volume.

\section{Laboratory assays}

PCT, CRP, IL-6, LBP, and sTREM-1 levels were determined according to the techniques previously described [13-15]. Results were expressed in microgram per liter $(\mu \mathrm{g} / \mathrm{l})$, milligram per liter (mg/l), picogram per milliliter ( $\mathrm{pg} / \mathrm{ml})$, microgram per milliliter $(\mu \mathrm{g} / \mathrm{ml})$, and picogram per milliliter $(\mathrm{pg} / \mathrm{ml})$, respectively.

Gas6 levels in postmortem serum were determined using a commercialized enzyme-linked immunosorbent assay (ELISA) kit according to manufacturer protocol. Results were expressed in picogram per milliliter.

\section{Statistical analysis}

Increased postmortem serum PCT, CRP, LBP, and sTREM-1 levels suggesting the presence of generalized inflammation and bacterial infections were chosen based on former medicolegal investigation results $(2 \mu \mathrm{g} / \mathrm{l}, 10 \mathrm{mg} / \mathrm{l}, 10 \mu \mathrm{g} / \mathrm{ml}$, and $90 \mathrm{pg} / \mathrm{ml}$, respectively). IL-6 cutoff value was set at $200 \mathrm{pg} /$ $\mathrm{ml}$, according to the findings reported by Llewelyn et al. [10].

Gas6 values in postmortem serum suggesting generalized inflammation and bacterial infections were not preliminarily identified due to the unavailability of previous studies on postmortem material for comparison.

A two-group comparison was performed nonparametrically by the Mann-Whitney $U$ test. $P$ values less than 0.05 were considered statistically significant. Spearman's rank correlation was used to evaluate the correlation between Gas6 and PCT in septic cases as well as between Gas6, IL-6, and CRP in the control cases. Graphpad Prism 4.0 (Graphpad Software, La Jolla, CA, USA) was used for statistics.

\section{Ethics}

All relevant ethical issues were identified and discussed with the local ethical committee. All cases collected for this study underwent medicolegal autopsies as requested by the public prosecutor. Postmortem serum from femoral blood is systematically collected in our facility prior to or during autopsy and biochemical investigations are routinely performed. All postmortem serum samples were anonymized prior to analysis. No further ethical approval was necessary to perform biochemical investigations in the cases included in the study herein.

\section{Results}

There were no gender-related or age-dependent differences in Gas6 levels in either sepsis or control cases. No correlation between Gas 6 concentrations and postmortem interval was observed in either sepsis or control cases. No correlation between Gas6 concentrations and survival time was noticed in the group of thoracic and/or abdominal trauma patients.

As expected, postmortem serum PCT, CRP, sTREM-1, IL6 , and LBP values were significantly higher in the sepsis group compared to the controls $(P<0.0001)$.

No statistically significant differences were found in postmortem serum Gas6 concentrations among sepsis, pulmonary embolism, trauma, or end-stage renal failure cases. Conversely, statistically significant differences were observed between hanging cases and the sepsis group $(P<0.0001)$ as well as between hanging cases and each of the other subcategories in the control group $(P<0.0001$ for each comparison).

In the sepsis group, a significant positive correlation was found between PCT and Gas6 concentrations $\left(r_{\mathrm{sp}}=0.63\right.$, $P<0.05$ ).

In the control group (pulmonary embolism, trauma, and end-stage renal failure cases), a significant positive correlation was found between Gas6 values and IL-6 levels $\left(r_{\mathrm{sp}}=0.59\right.$, $P<0.05)$ as well as between Gas6 values and CRP levels $\left(r_{\mathrm{sp}}=0.60, P<0.05\right)$.

These preliminary findings seem to indicate that Gas 6 can be effectively measured in postmortem serum from femoral blood obtained during autopsy. However, Gas6 levels in sepsis-related fatalities do not appear to be clearly distinguishable from concentrations in pulmonary embolism, severe trauma, and end-stage renal failure cases, at least as far as its determination in biological samples collected after death in cases originating from the forensic practice is concerned.

\section{Discussion}

Initial evidence suggesting involvement of Gas6 in modulating neutrophil functions and neutrophil-mediated host responses in inflammation and bacterial infection was reported by Borgel et al. [16]. These authors observed that severe sepsis and, to a lesser extent, patients with non-septic organ failure were characterized by increased plasma Gas 6 concentrations. In severe sepsis, Gas6 plasma levels correlated positively with the number of failing organs and disease severity. Moreover, within the sepsis cases, Gas6 concentrations were 
significantly higher in those with septic shock. Based on these findings, Borgel et al. [16] hypothesized that Gas6, through its anti-apoptotic function, might contribute to delaying neutrophil apoptosis at inflammation sites, thereby maintaining release of toxic granule components and production of reactive oxygen metabolites from neutrophils, both responsible for tissue injury and organ failure.

Gibot et al. [7] measured Gas6, sTREM-1, and PCT levels in a series of 45 septic shock patients and observed plasma Gas 6 concentrations ranging from 5 to $95 \mathrm{pg} / \mathrm{ml}$ upon admission (median $51 \mathrm{pg} / \mathrm{ml}$ ) to hospital. Positive correlations were noticed between acute renal failure and Gas6 levels as well as between hepatic dysfunction and plasma Gas 6 concentrations. A strong correlation was found between plasma PCT and Gas6 levels as well as between plasma sTREM-1 and Gas6 values. Conversely, no relation was identified between plasma Gas6 concentrations and other clinical or biological features, including gender, age, infection site, or microorganisms responsible for sepsis.

Based on the results obtained from in vivo and in vitro investigations, Tjwa et al. [8] speculated that Gas6 amplifies EC activation in response to inflammatory stimuli, accelerating circulating leukocyte and platelet sequestration. In addition, it promotes leukocyte extravasation, inflammation, and thrombosis in mouse models of endotoxemia, thus confirming a pivotal role for Gas6 in enhancing interactions between ECs and circulating cells during inflammation.

Ekman et al. [17] analyzed plasma concentrations of Gas6 and its soluble tyrosine kinase receptor sAxl in a series of subjects with severe sepsis, sepsis, infection without SIRS, SIRS without infection, and control cases. They observed that all patients had significantly increased plasma Gas6 concentrations compared to control cases, irrespective of the presence of infection, thus suggesting that Gas6 behaves as an acute phase protein. Additionally, patients with severe sepsis had significantly increased plasma Gas6 concentrations when compared to sepsis cases. Gas 6 concentrations correlated with those of IL-6, PCT, the number of failing organs, and disease severity in all patients considered together as well as in severe sepsis and sepsis patients evaluated separately. Moreover, in these latter, plasma Gas6 concentrations correlated inversely with CRP levels.

Gas6 has been demonstrated to be involved in vascular hemostasis in both animal and human studies. Gas6 null (-/-) mice have been shown to be protected from lethal venous and arterial thrombotic challenges, suggesting that Gas6 plays a prothrombotic role through defects in platelet aggregation [18-21]. More recently, Blostein et al. [18] provided the first evidence that elevated plasma Gas6 levels are associated with venous thromboembolic disease in humans.

Lastly, Lee at al. [2] found that Gas6 concentrations were significantly higher in patients with chronic kidney disease (CKD) compared to control individuals and postulated that dysregulation in the Gas6 pathway might contribute to vascular disease and chronic inflammation in renal failure.

Though further studies are required to confirm the preliminary findings herein stated, the results of our analyses seem to indicate that Gas6 is measurable in postmortem serum collected during autopsy. Moreover, as in the clinical setting, statistically significant differences in Gas6 concentrations were found between sepsis-related fatalities, fatal pulmonary embolism associated with deep vein thrombosis, end-stage renal failure patients, and control cases.

Table 1 Summary of ranges, medians, and mean values for all tested parameters in both studied groups

\begin{tabular}{|c|c|c|c|c|c|c|}
\hline & PCT $(\mu \mathrm{g} / \mathrm{l})$ & $\mathrm{CRP}(\mathrm{mg} / \mathrm{l})$ & sTREM-1 (pg/ml) & IL-6 (pg/ml) & $\mathrm{LBP}(\mu \mathrm{g} / \mathrm{ml})$ & Gas6 (pg/ml) \\
\hline \multirow[t]{3}{*}{ Sepsis $(n=24)$} & $2.16-7.65$ & $66-250$ & $60-340$ & $540-3600$ & $14-68$ & $190-356$ \\
\hline & $(3.91)$ & $(106)$ & $(160)$ & $(1150)$ & $(26)$ & $(225)$ \\
\hline & $(4.06)$ & $(120)$ & $(180)$ & $(1290)$ & $(32)$ & $(240)$ \\
\hline \multirow[t]{3}{*}{ Pulmonary embolism $(n=6)$} & $0.06-0.18$ & $16-29$ & $30-80$ & $140-450$ & $6-12$ & $170-343$ \\
\hline & $(0.08)$ & $(20)$ & $(48)$ & $(330)$ & (7) & $(210)$ \\
\hline & $(0.09)$ & $(22)$ & $(45)$ & $(280)$ & (8) & $(220)$ \\
\hline \multirow[t]{3}{*}{ Trauma $(n=6)$} & $0.10-0.21$ & $21-34$ & $50-140$ & $160-380$ & $10-19$ & $180-332$ \\
\hline & $(0.12)$ & $(26)$ & (75) & $(240)$ & $(12)$ & $(215)$ \\
\hline & $(0.14)$ & $(28)$ & $(110)$ & $(280)$ & $(15)$ & $(222)$ \\
\hline \multirow[t]{3}{*}{ End-stage renal failure $(n=6)$} & $0.42-0.66$ & $26-45$ & $75-220$ & $200-410$ & $12-22$ & $166-340$ \\
\hline & $(0.48)$ & $(30)$ & (120) & $(260)$ & (16) & (206) \\
\hline & $(0.52)$ & (36) & (136) & $(320)$ & (18) & (216) \\
\hline \multirow[t]{3}{*}{ Hanging $(n=6)$} & $0.06-0.14$ & $4-9$ & $20-59$ & $30-180$ & $5-10$ & $60-170$ \\
\hline & $(0.08)$ & $(5)$ & (32) & $(60)$ & (6) & $(85)$ \\
\hline & $(0.08)$ & (6) & (36) & (90) & (7) & (90) \\
\hline
\end{tabular}

Abbreviations are reported in the text 
We did not find statistically significant differences in postmortem serum Gas6 levels among sepsis-related deaths, pulmonary embolism, severe trauma, or chronic kidney disease cases. This would indicate that Gas 6 can be considered a general marker of inflammatory conditions rather than a specific biomarker of sepsis. Indeed, as emphasized by some researchers, this possible role of Gas6 would fit well with the view of the TAM receptor system as a brake for innate immunity. Gas6 itself has been shown to have anti-inflammatory properties in certain cells, reducing or inhibiting TNF- $\alpha$ and IL-6 secretion from activated macrophages, while it could also orchestrate the course of inflammation by favoring platelet and leukocyte interactions with the endothelium [3, 22, 23].

It must also be stressed that range, median, and mean Gas6 values in our sepsis cohort (Table 1) were markedly higher than those described by Gibot et al. [7] in living patients with septic shock on admission to hospital. In this study, nonsurviving patients exhibited persistently elevated Gas6 levels, whereas survivors were characterized by progressively declining Gas6 concentrations, especially after day 7.

These results are more difficult to explain. It can be hypothesized that the markedly increased Gas6 levels found in our study in sepsis-related fatalities are the expression of the extremely severe organ dysfunction occurring shortly prior to death. On the other hand, the significantly elevated Gas6 concentrations observed in our sepsis and control cohorts compared to the values measured in living patients with septic shock could also represent the result of postmortem leakage from damaged or necrotic ECs.

Though we did not observe any correlation between Gas6 levels and the postmortem interval in either sepsis or control cases, it cannot be excluded that rapid cell membrane breakdown after death due to decompositional changes can favor and maintain the molecule's release into the bloodstream. This has already been described for other biomarkers measured in samples collected at autopsy [24, 25].

The limitations of our study must be acknowledged. The first is the relatively small number of subjects, which may limit the accuracy of the research. However, precise selection criteria were applied during sample collection. Moreover, the recruitment process was rigorous in all groups of patients in order to minimize heterogeneity in the study populations. Secondly, detailed studies on the behavior of Gas6 in the postmortem setting are unavailable at present, making our observations mostly speculative. Prospective studies including a greater number of subjects are therefore needed to substantiate or invalidate our findings.

To conclude, the study herein presented is the first assay of measuring Gas6 values in postmortem samples in septic and control cases that underwent medicolegal investigations. Even though further analyses are required to confirm these preliminary findings, our data seem to indicate that Gas6 is measurable in postmortem samples. However, since Gas6 levels in sepsis-related fatalities do not appear to be clearly distinguishable from concentrations in other situations characterized by generalized inflammation, its diagnostic value in identifying sepsis-related deaths in forensic pathology routine remains limited at present.

Conflict of interest The authors declare that they have no competing interests.

Funding source This study was not financially supported.

\section{References}

1. Uehara S, Handa H, Gotoh K, Tomita H, Sennshuu M (2009) Plasma concentrations of growth arrest-specific protein 6 and protein $\mathrm{S}$ in patients with acute pancreatitis. J Gastroenterol Hepatol 24:1567-73

2. Lee IJ, Hilliard B, Swami A, Madara JC, Rao S, Patel T, Gaughan JP, Lee J, Gadegbeku CA, Choi ET, Cohen PL (2012) Growth arrest-specific gene 6 (Gas6) levels are elevated in patients with chronic renal failure. Nephrol Dial Transplant 27:4166-72

3. Alciato F, Sainaghi PP, Sola D, Castello L, Avanzi GC (2010) TNFalpha, IL-6, and IL-1 expression is inhibited by GAS6 in monocytes/macrophages. J Leukoc Biol 87:869-75

4. Fiebeler A, Park JK, Muller DN, Lindschau C, Mengel M, Merkel S, Banas B, Luft FC, Haller H (2004) Growth arrest specific protein 6/Axl signaling in human inflammatory renal diseases. Am J Kidney Dis 43:286-95

5. Hung YJ, Lee CH, Chu NF, Shieh YS (2010) Plasma protein growth arrest-specific 6 levels are associated with altered glucose tolerance, inflammation, and endothelial dysfunction. Diabetes Care 33:1840-4

6. Park JK, Theuer S, Kirsch T, Lindschau C, Klinge U, Heuser A, Plehm R, Todiras M, Carmeliet P, Haller H, Luft FC, Muller DN, Fiebeler A (2009) Growth arrest specific protein 6 participates in DOCA-induced target-organ damage. Hypertension 54:359-64

7. Gibot S, Massin F, Cravoisy A, Dupays R, Barraud D, Nace L, Bollaert PE (2007) Growth arrest-specific protein 6 plasma concentrations during septic shock. Crit Care 11:R8

8. Tjwa M, Bellido-Martin L, Lin Y, Lutgens E, Plaisance S, Bono F, Delesque-Touchard N, Hervé C, Moura R, Billiau AD, Aparicio C, Levi M, Daemen M, Dewerchin M, Lupu F, Arnout J, Herbert JM, Waer M, García de Frutos P, Dahlbäck B, Carmeliet P, Hoylaerts MF, Moons L (2008) Gas6 promotes inflammation by enhancing interactions between endothelial cells, platelets, and leukocytes. Blood 111:4096-105

9. Que YA, Delodder F, Guessous I, Graf R, Bain M, Calandra T, Liaudet L, Eggimann P (2012) Pancreatic stone protein as an early biomarker predicting mortality in a prospective cohort of patients with sepsis requiring ICU management. Crit Care 16:R114

10. Llewelyn MJ, Berger M, Gregory M, Ramaiah R, Taylor AL, Curdt I, Lajaunias F, Graf R, Blincko SJ, Drage S, Cohen J (2013) Sepsis biomarkers in unselected patients on admission to intensive or highdependency care. Crit Care 17:R60

11. Palmiere C, Augsburger M (2014) Endocan measurement for the postmortem diagnosis of sepsis. Leg Med (Tokyo) 16:1-7

12. American College of Chest Physicians/Society of Critical Care Medicine Consensus Conference (1992) Definition for sepsis and organ failure and guidelines for the use of innovative therapies in sepsis. Crit Care Med 20:864-74 
13. Palmiere C, Bardy D, Mangin P, Augsburger M (2013) Value of sTREM-1, procalcitonin and CRP as laboratory parameters for postmortem diagnosis of sepsis. J Infect 67:545-55

14. Augsburger M, Iglesias K, Bardy D, Mangin P, Palmiere C (2013) Diagnostic value of lipopolysaccharide-binding protein and procalcitonin for sepsis diagnosis in forensic pathology. Int $\mathrm{J}$ Legal Med 127:427-35

15. Schrag B, Roux-Lombard P, Schneiter D, Vaucher P, Mangin P, Palmiere C (2012) Evaluation of C-reactive protein, procalcitonin, tumor necrosis factor alpha, interleukin-6, and interleukin-8 as diagnostic parameters in sepsis-related fatalities. Int J Legal Med 126: 505-12

16. Borgel D, Clauser S, Bornstain C, Bièche I, Bissery A, Remones V, Fagon JY, Aiach M, Diehl JL (2006) Elevated growth-arrestspecific protein 6 plasma levels in patients with severe sepsis. Crit Care Med 34:219-22

17. Ekman C, Linder A, Akesson P, Dahlbäck B (2010) Plasma concentrations of Gas6 (growth arrest specific protein 6) and its soluble tyrosine kinase receptor $\mathrm{sAxl}$ in sepsis and systemic inflammatory response syndromes. Crit Care 14:R158

18. Blostein MD, Rajotte I, Rao DP, Holcroft CA, Kahn SR (2011) Elevated plasma gas6 levels are associated with venous thromboembolic disease. J Thromb Thrombolysis 32:272-8
19. Laurance S, Lemarié CA, Blostein MD (2012) Growth arrest-specific gene 6 (gas6) and vascular hemostasis. Adv Nutr 3:196-203

20. Robins RS, Lemarié CA, Laurance S, Aghourian MN, Wu J, Blostein MD (2013) Vascular Gas6 contributes to thrombogenesis and promotes tissue factor up-regulation after vessel injury in mice. Blood 121:692-9

21. van der Meer JH, van der Poll T, Van 't Veer C (2014) TAM receptors, Gas6, and protein S: roles in inflammation and hemostasis. Blood 123:2460-9

22. Hurtado B, de Frutos PG (2010) GAS6 in systemic inflammatory diseases: with and without infection. Crit Care 14:1003

23. Rothlin CV, Ghosh S, Zuniga EI, Oldstone MB, Lemke G (2007) TAM receptors are pleiotropic inhibitors of the innate immune response. Cell 131:1124-36

24. Palmiere C, Mussap M, Bardy D, Cibecchini F, Mangin P (2013) Diagnostic value of soluble CD14 subtype (sCD14-ST) presepsin for the postmortem diagnosis of sepsis-related fatalities. Int J Legal Med 127:799-808

25. Palmiere C, Augsburger M, Mangin P (2014) High-mobility group box-1 protein determination in postmortem samples. Forensic Sci Int 239:103-6 